\title{
The impact of transit signal priority: case study in Dubai, UAE
}

\author{
Hadeel Al Khateeb ${ }^{1 *}$ and Akmal Abdulfatah ${ }^{2}$ \\ ${ }^{1}$ Director of Planning \& Survey Dept. - Ajman Municipality, Ajman - UAE \\ ${ }^{2}$ Associate Professor, Civil Engineering - American University of Shrajah, Sharjah - UAE
}

\begin{abstract}
Abdulfatah \& Alkhateeb proposes on this paper one of the Intelligent Transportation Systems- ITS, the Transit Signal Priority- TSP, which involves real time tracking. The United States is considered one of the lead countries in implementing this strategy since decades ago. Europe and Germany have also implemented TSP quite extensively to improve rider-ship and buses efficiency. TSP is a strategy that supports the transit system through easing the movement of the service. The operational technique used for TSP is controlling the signal system at the intersections. A detector system must be integrated in the implementation stage. It will detect the preference of public buses among the other vehicles in the traffic queue. Determining of optimal detector location for the TSP with queued lanes is the main feature. It must be studied and decided on before starting different signalization proposals for the intersection. Controlling traffic signal in favor of the public bus system will improve reliability and efficiency for buses. Meanwhile, the extreme impact of the TSP on general traffic as well as on public buses became the basis for many recent studies and thesis tested by many authors. The scope of the project as addressed in this paper is to report the delivered results of different scenarios tested, and then compare these proposed scenarios to select the best signal consideration, which has a potential impact on bus and traffic performance. After applying TSP, the results show that the impact of actuating traffic signals in Al Mussallah / Al Maktoum corridor after extending green time for bus approach $(15 \mathrm{sec})$ through minimum reduction on average travel time for busses by $7.04 \%$ and tremendous increase in travel time for cars by $38.68 \%$. A reduction in cars Avg. Speed by $28.04 \%$ in comparison to $35.52 \%$ buses Avg. Speed. On the other hand, a reduction in buses delay received by $14.11 \%$ and a decrease in Avg. Stopped delay per bus of about $18.56 \%$, while cars received an increase in delay of around $57.00 \%$ with increased Avg. Stopped delay per car of $55.13 \%$.
\end{abstract}

\footnotetext{
*Corresponding author: Hadeel.akhateeb@gmail.com
} 


\section{Introduction}

In the last three decades, the demand for using different modes of transportation in many countries became a tremendous need. The main objective of many governmental transport authorities all over the world is to supply and/or improve different transport services to accommodate the increasing demand for daily travels. Accordingly, recommending new strategies and/or replicating the most successful practices based on travelers' preferences became a target. Implementing and improving road network infrastructure is also recommended as it encourages more efficient and reliable transit system operations in the city. All these goals require policies/ strategies and need to be operated through implementation plans.

This paper proposes one of the Intelligent Transportation Systems- ITS, the Transit Signal Priority- TSP, which involves real time tracking. The United States is considered one of the lead countries in implementing this strategy since decades ago. Europe and Germany have also implemented TSP quite extensively to improve rider-ship and buses efficiency.

\subsection{TSP considerations}

The TSP is designed to benefit the public transport (buses) in the signalized intersections. If it is successful, then the impact will be reflected on the buses performance, and will increase the service reliability and efficiency. Thus, it will improve travel time on overall traffic. Accordingly, surrounding traffic environment must be considered when implementing TSP in any signalized intersection. TSP in brief has been proven through many case studies all over the world to have either positive/negative impact on the traffic stream or both on some cases. Both cases happen when assuming that we have Red truncation (Early green priority) strategy (Garrow, M. and Machemehl 1998, and A. Skabardonis, 2000) [1] which was in favor of bus approach through early green; before the completion of the full signal cycle for the whole system. As a result, the early green for the detected buses will be improved compared to opposite directions which will receive more delays and lead to vehicles queue.

Advanced control systems and the new Auto Vehicle Identification system - AVI, which are available in the market nowadays, have been incorporated in the TSP strategy to achieve the successfulness (Strategies for Transit: A Review of Selected Experiences in the United States, The Seventh World Congress on Intelligent Transportation Systems, 2000) [5]. Criteria demanded when implementing TSP:

- Vehicle volumes other than buses (Vehicular volume)

- Traffic signal coordination

- Bus headways and volumes (frequency and occupancy)

- Bus stops location (i.e. near v/s far-side traffic signal)

- Pedestrian

\subsubsection{Bus stops}

As per recorded in the TSP planning and Implementation Handbook, and the results of the surveys conducted by different Transportation Agencies, it is most preferable to locate all bus stops on far- side; as it reduces the green extension interval that is required to be granted to the bus. (Manual of Uniform Traffic Control Devices, 2000) [4]. It minimizes the difficulty when signal controller is predicting the bus arrival. Accordingly, it will give more accuracy for the prediction and will facilitate the operation of bus checking out of the 
system. (Transit Signal Priority System Performance Monitoring and Optimization, 2001) [8]. Figure (1) illustrates the two different bus stop types.

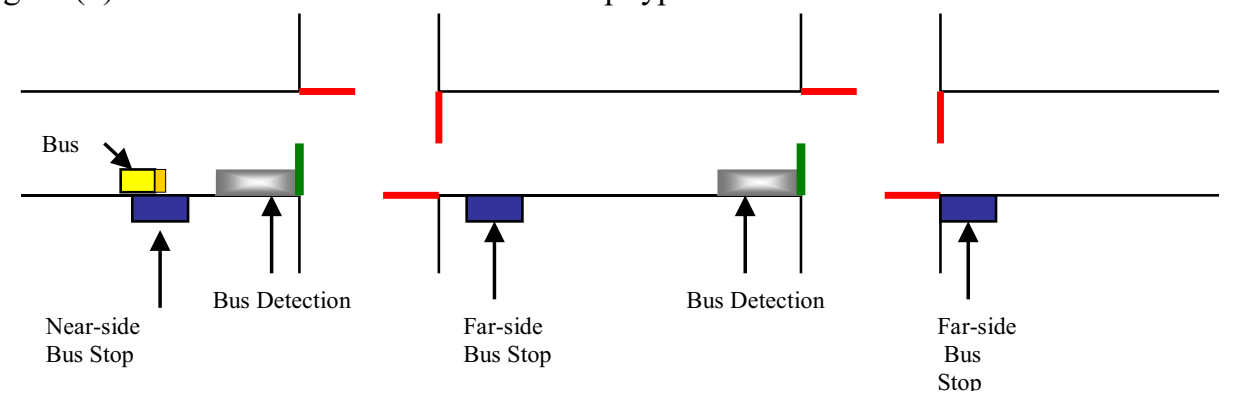

Fig. 1. Location of Bus Stops and Distance from TSP Detector.

\subsubsection{Pedestrians}

TSP strategy offers special treatment for transit vehicles (buses) crossing a signalized intersection. However, some challenges need to be studied/ considered when implementing TSP. Since most of signalized intersections, which will become TSP signalized intersections, have at the same time special signals for pedestrians. The pedestrian signals are always timed to automatically offer pedestrian pass signal (Green light in their special designed signals). The time of this green is to be consistent with the Red Truncation of the same vehicles signals, to allow them safely crossing in the street. This issue is still not extensively discussed since it is fully dependent on the pedestrian volume at the time the bus reaches the detection zone. (A. Skabardonis, Paper Presented at the 79th Annual Meeting of Transportation Research Board, 2000) [3].

\subsection{TSP route characteristic}

The best route for implementing TSP application is where the road is operating under multi-modal traffic system with Four to Six Lanes in Arterials. Currently, minimum number for routes deploying TSP in the US are Two and few cities have Six Routes and above and it will increase in the future. In North America, Bus Transit Signal Priority- BRT is deployed for only Buses/ BRT. Both concepts, TSP and BRT, are two faces for the same coin. However, Transit vehicles are those vehicles in service (The Seventh World Congress on Intelligent Transportation Systems, Turin, Italy, 2000) [4]. In some other cities, such as Philadelphia, the streetcar system operates on regular streets with other traffic vehicles. While Light Rail Transit - LRT application is implemented on dedicated Right-of-Way ROW separate from other traffic vehicles (Collura, J. and others, 2001). [7].

The number of signalized intersections varies between the two-utilizing left-turn phase to more than 1,500 intersections in large metropolitans (i.e. Houston has 1563). Bus stops maybe located either near the intersection or far away from it. Meanwhile, it is preferable to have the bus stop far away from the intersection; this would help to more accurately predict the arrival time for the transit vehicle at the intersection. Thus, it will lead to a reduction in the green extension (i.e. Transit vehicle checking out). When route is operating under TSP, the headway will range from 90 - seconds to 30 -minutes. But the peak hour headway ranges from 5- minutes to 30- minutes according to the route and service type, according to the Manual of Uniform Traffic Control Devices, 2000) [4]. 


\subsubsection{Bus transit lines}

At this paper, a Transit line is defined as the Bus Transit Lines designed to serve buses or Transit Vehicles. It is a sequence of multiple transit stops fixed according to a specified time table (Bill Kloos, 2002) [9]. The determination of the stop times is basically made by dwell time distributions or according to the service times for the passengers. By the time of drafting this paper all the stated lines were operated by RTA, Bus Operation section must verify the location of the new bus stops, code the bus lines on each route, and add accumulated bus frequencies (bus per peak hour per direction). When using VISSIM similarly to the private vehicles generated volumes, the transit line codes must be entered by external station per route not the arrival distribution.

TSP strategy requires 'Signal controller' to change the traffic light and 'Confirmation device' to allow traffic movement in the intersection (Figure 2). An operation traffic signal is dictated by traffic signal system capability. Traffic signals are designed to operate in a way to minimize total traveler delay. In other words, the traveler delay is eventually the transit vehicle delay as the case of TSP (A. Skabardonis, 2000) [3].

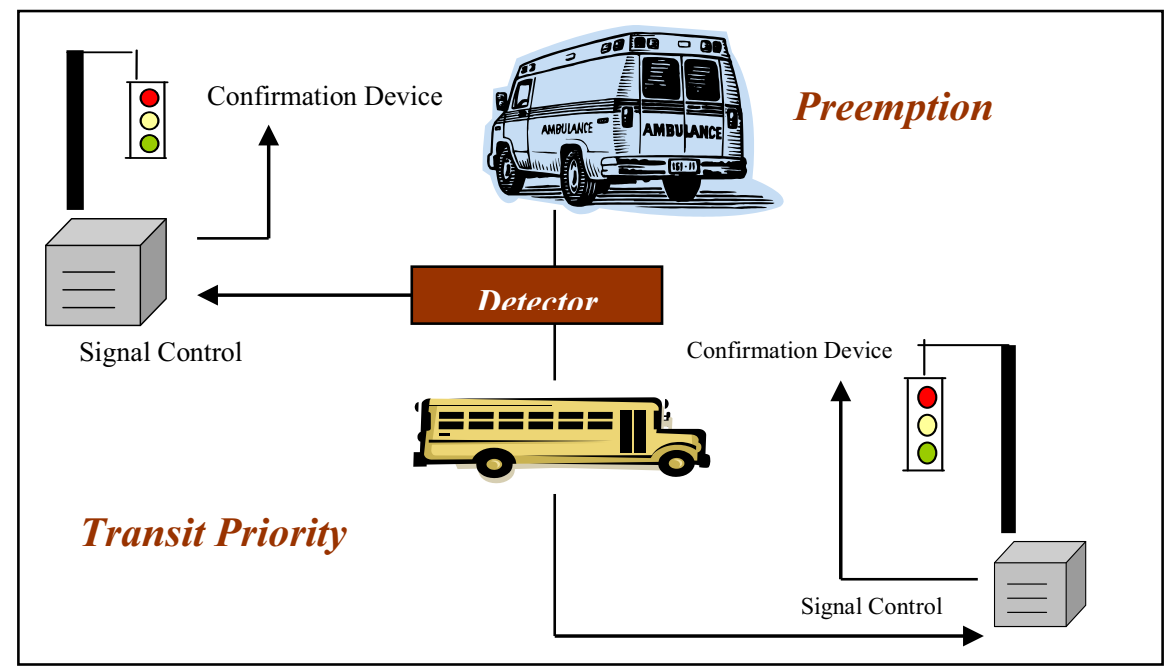

Fig. 2. Example of the transit and preemption operation.

\subsubsection{Waiting Time}

Waiting time is a component specified for only public transport mode. It is based on the frequency of the public buses available for travel, according to the Final Report, prepared to St. Cloud Metropolitan Transit Commission, Minnesota, 2000[6]. The bus users are either considered to have no idea about the timetable and may arrive at any time at a bus stop and wait for the bus arrival, or they may have a reasonable idea about bus timetables. The majority are considered to be from the second type of bus users/ passengers. The regular passengers are most likely to reach the bus stop within a reasonable time limit before bus services arrival. The following formula is showing how the waiting time is calculated for those passengers: 


$$
W T= \begin{cases}\frac{\text { Headway }}{2} & \text {, if Headway } \leq 9 \text { minutes } \\ 1.5 x^{\text {Headway }} & \text {, if Headway } \geq 9 \text { minutes }\end{cases}
$$

Where, WT is the waiting time

Bus Headway: is the amount of time (usually in minutes) when buses are passing certain point on lane in a successive manner. This point is measured by a reference point on the buses, according to the Manual of Uniform Traffic Control Devices, 2000[4].

\section{Literature review}

In the last three decades, the demand for using different modes of transportation in many countries became a tremendous need. The main objective of many governmental transport authorities all over the world is to supply and/or improve different transport services to accommodate the increasing demand for daily travels. Accordingly, recommending new strategies and/or replicating the most successful practices based on travelers' preferences became an essential target. Improving roads infrastructure is another means to encourage more efficient and reliable transit system operations. All these goals require multiple policies on a strategic level, and need to be operated through implementation plans.

This project proposes one of the Intelligent Transportation Systems- ITS, the Transit Signal Priority- TSP, which involves real time tracking. The United States is considered one of the lead countries in implementing this strategy since decades ago. Europe and Germany have also implemented TSP quite extensively to improve rider-ship and buses efficiency. This is as per stated in the report issued by Transportation Research Board of National Academies, Bus and Rail Transit Preferential Treatments in Mixed use Traffic, $2010[16]$.

\subsection{TSP considerations}

The TSP is essentially designed to benefit the public transport (buses) in the signalized intersections. If this is achieved successfully, then the impact will be reflected on the buses performance, and will increase the service reliability and efficiency. On the other hand, the system performance will impact the travel time on overall traffic. Accordingly, the surrounding traffic environment must be considered when implementing TSP in any signalized intersection. Hereon, traffic considerations will directly impact the overall traffic conditions and are supposed to be analyzed to evaluate the impact of the TSP. TSP in brief is as proven through many case studies all over the world to have either positive or negative impact on the traffic stream or both in some cases. The question that could be raised is when these two extremes "Negative and Positive" happen at the same time. The answer is clearly justified as follows: by assuming that we have Red truncation (Early green priority) strategy (Garrow, M. and Machemehl 1998, and A. Skabardonis, 2000) [1]:

- When bus passes by the detection zone then the detector will distinguish between it and other vehicles passing the same corridor.

- The detector will send certain algorithms to the signal controllers.

- The signal controller will translate the received algorithms to actuated parameters for each signal in the intersection 
- In the corridor, the approach of the detected bus will be receiving the signal priority, which is in this case the early green before the completion of the full signal cycle for the whole system in the corridor.

- Thus, the signal will be turned to green as the bus approaches while the approach that is supposed to get the green signal will receive expansion in the red truncation which will increase the delay on that approach.

- As a result, the early green for the detected bus approach will be the fortunate direction. While the opposite directions (any of the other approaches), will receive extended red signal timing which will cause more delay on these directions and lead to vehicles queue.

High advanced control systems and the new Auto Vehicle Identification system - AVI, which are available in the market nowadays, have been incorporated in the TSP strategy to achieve the successfulness (Strategies for Transit: A Review of Selected Experiences in the United States, The Seventh World Congress on Intelligent Transportation Systems, 2000) [5]. Criteria demanded when implementing TSP:

- Vehicle volumes other than buses (Vehicular volume)

- Traffic signal coordination

- Bus headways and volumes (frequency and occupancy)

- Bus stop locations (i.e. near v/s far-side traffic signal)

- Pedestrians

\subsection{TSP benefits and impacts - case studies}

Implementing TSP will generate multiple benefits for the city if it decides to adopt the ITS strategy. Many countries tend to implement it because of the following general impacts such as reduction in: travel time for Transit vehicles, Transit delay, stops, and schedule unreliability. There are minor impacts to cross street traffic and buses. Bearing in mind the impact of the TSP is difficult to be measured when the difference between before and after scenarios are small. Thus, TSP impact is approached when higher passengers and operational benefits for the Transit vehicles tempt to become faster, and when there is less variable travel time and improved on-time performance (Ghada, S. and Newland, 2002) [10].

Although, controlling before/after TSP condition requires good knowledge and understanding of the traffic condition in the targeted corridor, it is still difficult to measure the impact when the following variations appear in the study corridor (i.e. traffic volume changes, traffic accidents, weather weekends, and arrival time for the data collected by personnel. Many studies tested the impact of turning on/off the TSP for the sake of gathering information and studying the impact by comparing the outcome. These experiments impact transit vehicle drivers negatively, since they usually benefit from this strategy. The whole experiment was not ideal. But the simulation could predict the impact through testing different scenarios which is summarized in 5 published case studies, justifying the general impact of the TSP applications on Buses performance. (Bill Kloos, 2002) [9]. 


\section{Methodology}

\subsection{Project scope}

Multiple site visits were carried out to record observations and to understand all measures related to system operations of TSP by 2007. Some interview protocols, and some findings commissioned by RTA pervious relevant studies such as Bus Priority Study. Useful information about the new proposals introduced by PTA as a short- term strategies.

The scope of the project is to analyze the impact of the Transit Signal Priority (TSP) application using VISSIM software. The project is considered the Bus Route in Deira Area which will connect "Al Mussalla Street - 15" with "Al Maktoum Road - 12" to implement the proposed TSP. This corridor will be evaluated according to many reasons such as its mixed-use area and the corridor lies in the CBD area and it includes (4) signalized intersections, where the TSP can be implemented (Figure -3)

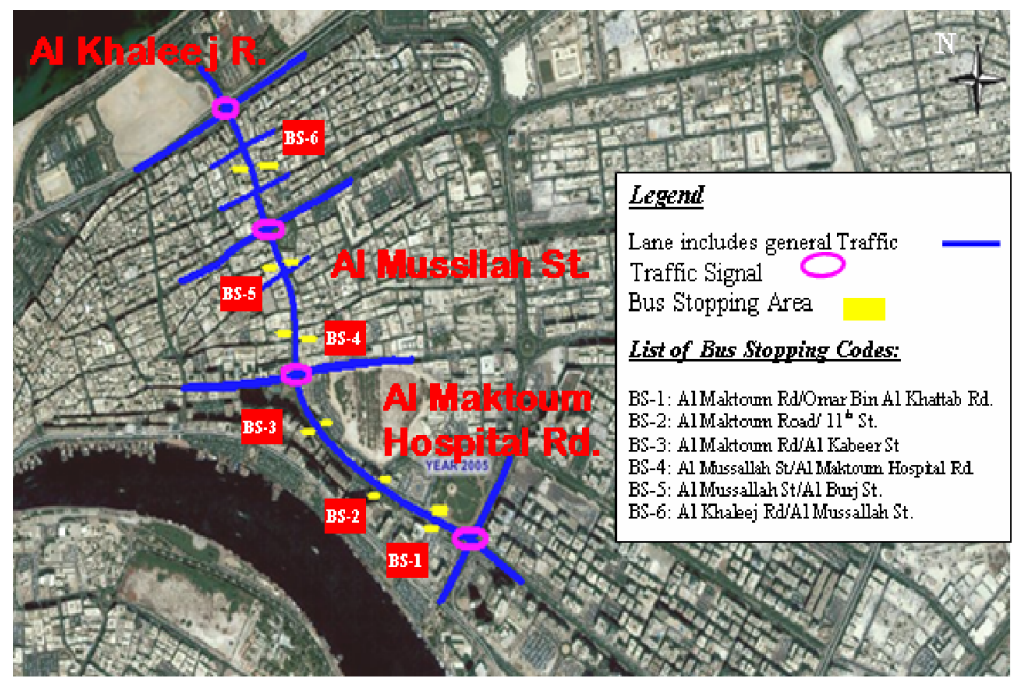

Fig. 3. Al Mussallah /Al Maktoum Corridor.

\subsection{Project methodology}

The project designed to evaluate the impact of TSP on the study corridor and tent to evaluate the impact of it on the (Buses) versus (Private vehicles) crossing this corridor in 3 main traffic conditions (Morning Peak-AM, Lunch Peak- LT, and Evening Peak - PM). As per Dubai R-1000 model, it is specified as short-term (2010) - all the proposed scenarios will be tested and analyzed for the short term horizon, medium term (2015), and target year (2020). Volumes extracted from R-1000 Dubai transportation model and (Road capacity, Public transport lines, Buses stops, Alignments, Headways) in accordance with the future policies, network improvements, and metro operation, the results expected to be not much differing from the analysis of the short term period - 2010. Adopted methodology to develop TSP scenarios was through evaluating the impact of the TSP by using VISSIM on different traffic conditions for the different horizons; through comparing results among different scenarios for Buses versus private vehicles, in order to recommend choice for the TSP strategy. 
RTA previous Mass Transit Plan for Dubai (PS - 700) 1997, (PS- 002) 1999, (PS 007) 2000, and (R-1000) 2005, and Public Transport Bus Master Plan were considered the bases for data collections. The methodology also relied on the compilation of data from secondary sources such as the updated (VISUM- 9.4) model [12], run (R-1000) model for both public transport and private vehicles and extracting volumes from the model. In addition; collected data for Public transport existing routes and networks, conducted interviews with public transport market stakeholders. Using micro-simulation model "VISSIM- 9.4" [13] a software tool to experience the different scenarios. Synchro [11] is a signalized model and is used to extract the signals coordination for the fixed- time signals to be used in the VISSIM. Finally, VisVAP [14] which is the flow chart program which includes multiple loop statements with specified functions has been used in order to implement the TSP. Which in turn is required to create (*.PUA) [15] file for inter-stages descriptions that are considered the parameters and the definitions which are all referred by the VisVAP in order to actuate the program as illustrated in Figure 4.

All stated tools presented the impact of the different signal timings on different measures:

- Average mean speed

- Travel time

- Delay

- Network performance

- Queue length

- Number of stops, and

- $\quad$ Link evaluation

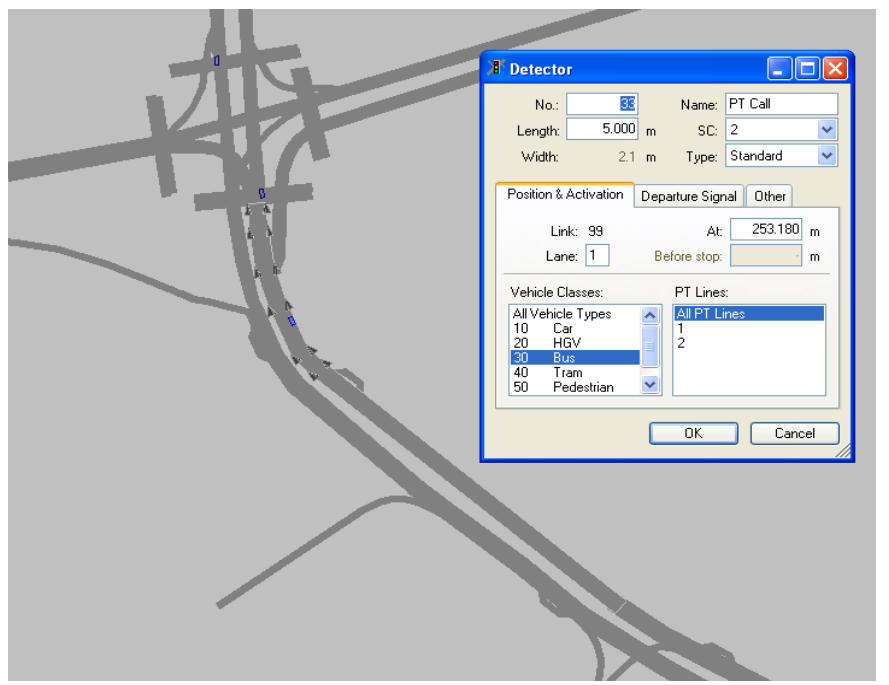

Fig. 4. Model Simulated through VISUM/ VISSIM/ VIS VAP.

\subsection{Experimental design}

Accordingly, the project final design is summarized in figure (5). 


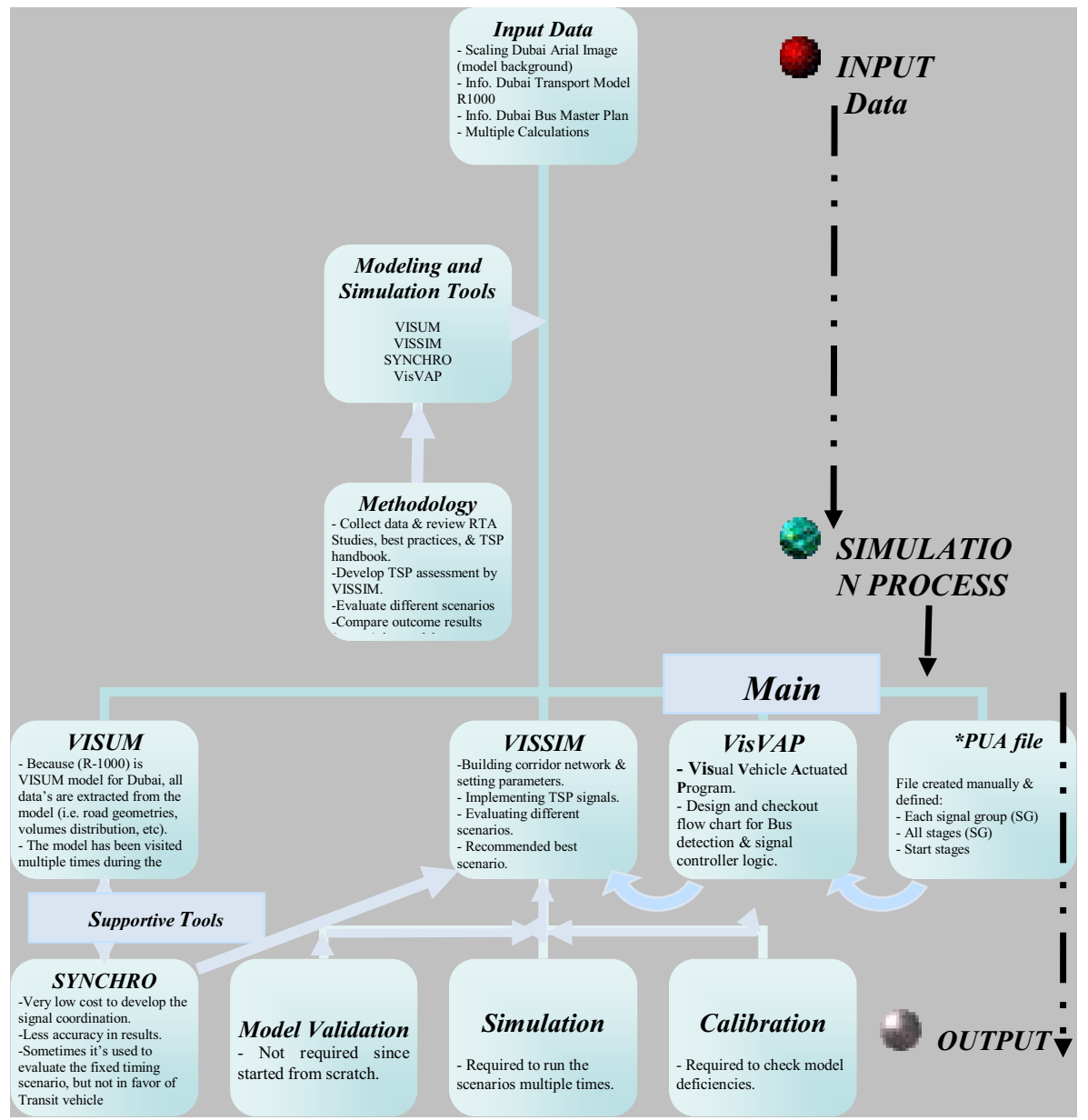

Fig. 5. Experimental Technical Design.

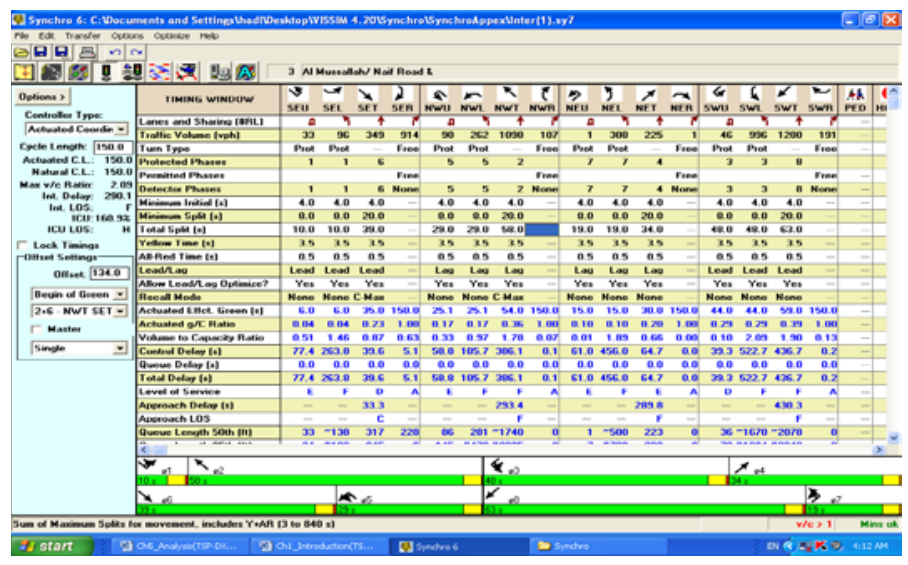

Fig. 6. Green Extension Method is considered in the Evaluation. (Syncro 6). 


\section{Results and discussion}

Developing the U-turn adjustment factor requires to model the relation between the percentage of UT traffic and the average discharge headway. For each site, four regression models were attempted: Linear, Logarithmic, Quadratic, and Exponential.

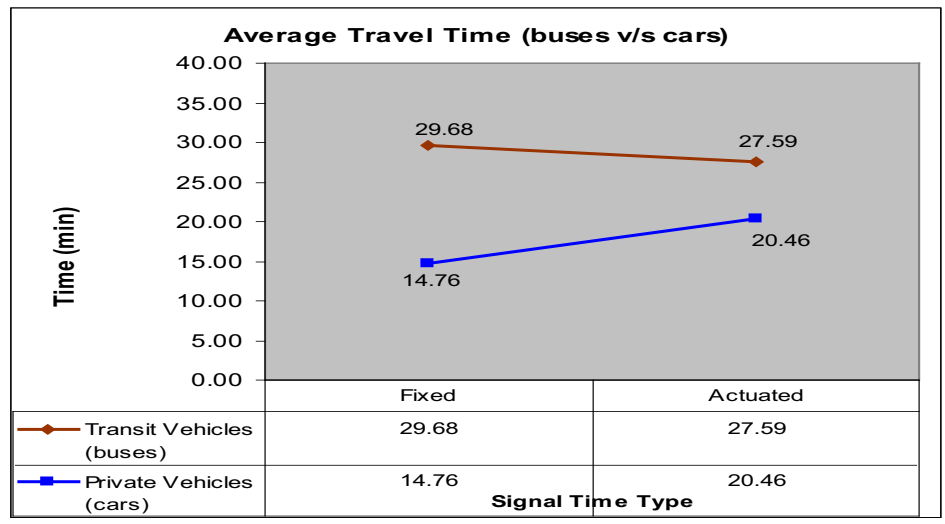

Fig. 7. Measurements Analysis.

After analysis implementation as illustrated in Figure 7, the results of the AM_2010 peak for both Fixed \&Actuated time scenarios were analyzed after simulating/ running the model 5 times under different seeds to reach consistent results. LT_2010 and PM_2010 peaks were also simulated but not analyzed since the results were too close to the AM_2010. Tables (1-2-3-4) shows the results of Fixed and Actuated scenario in favor of Buses.

Table 1. Average Travel Time Rate of Change.

\begin{tabular}{|c|c|c|c|}
\hline Vehicle Type & Fixed (min) & Actuated (min) & Rate of Change \\
\hline Private Vehicle (Car) & 14.76 & 20.46 & 38.68 \\
\hline Transit Vehicle (Bus) & 29.68 & 27.59 & -7.04 \\
\hline
\end{tabular}

Table 2. Average Speed Rate of Change.

\begin{tabular}{|c|c|c|c|}
\hline Vehicle Type & Fixed (Kph) & Actuated (kph) & Rate of Change \\
\hline Private Vehicle (Car) & 6.622 & 4.765 & $-28.04 \%$ \\
\hline Transit Vehicle (Bus) & 7.735 & 10.482 & $35.52 \%$ \\
\hline
\end{tabular}

Table 3. Average Delay Rate of Change.

\begin{tabular}{|c|c|c|c|}
\hline Vehicle Type & Fixed (s) & Actuated (s) & Rate of Change \\
\hline Private Vehicle (Car) & 426.889 & 670.212 & $57.00 \%$ \\
\hline Transit Vehicle (Bus) & 695.162 & 597.042 & $-14.11 \%$ \\
\hline
\end{tabular}

Table 4. Average Stopped Delay Rate of Change.

\begin{tabular}{|c|c|c|c|}
\hline Vehicle Type & Fixed & Actuated & Rate of Change \\
\hline Private Vehicle (Car) & 678.592 & 1052.679 & $55.13 \%$ \\
\hline Transit Vehicle (Bus) & 478.344 & 389.560 & $-18.56 \%$ \\
\hline
\end{tabular}


Figures ( 8 and 9) with Table (5) represent the queue lengths in general is getting higher when using actuated signals especially in the last 4 sections in the link. Whereas the queue counters number 1, 2, 3, 6, and 7 implemented in the bus approaches are increased. However, queue counters number 4,5 , and 8 show reductions in the queue length after treating bus approaches with the green extension. The logical reason is because the average queue length is already very high when the transit vehicle (bus) is detected by the check-in detector in order to start its treatment (green extension). Basically, the queue already started since this sub network is a part of the whole Deira road network, which means it is impacted by previous queues occurring due to other interchanges/ intersections connected to the corridor. Moreover, the scenario is testing the peaks period where the vehicle volumes is the highest. It is to be expected that in the off peak period the queue lengths will be less which will lead to a reduction in the number of stops within the queue.

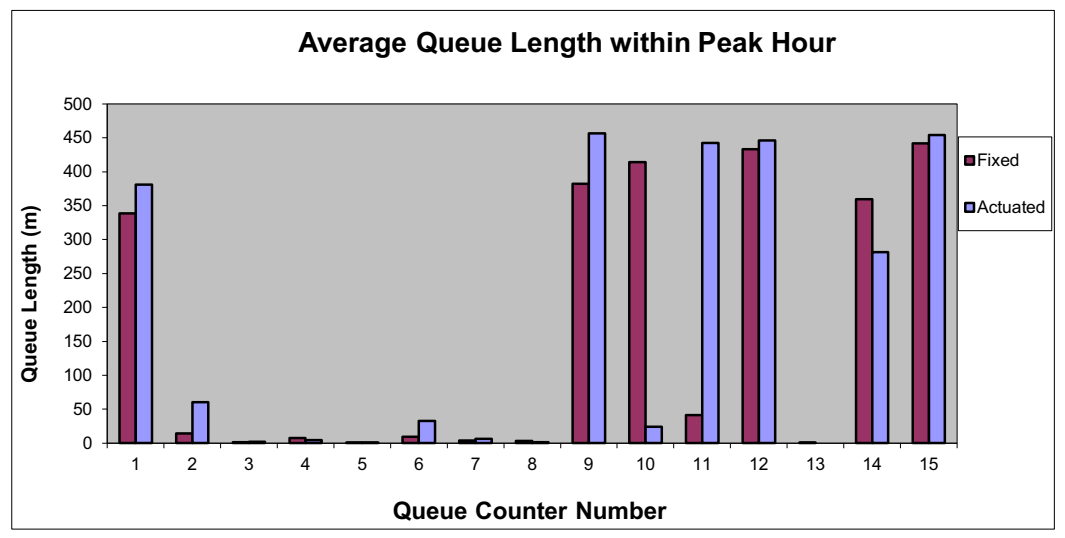

Fig. 8. Average Queue Length within Peak Hour.

Table 5. Average queue length (fixed $\mathrm{v} / \mathrm{s}$ actuated).

\begin{tabular}{|c|c|c|c|}
\hline QC & Fixed $(\mathrm{m})$ & Actuated $(\mathrm{m})$ & Rate of Change \\
\hline 1 & 339 & 381 & 12.60 \\
\hline 2 & 14 & 61 & 323.26 \\
\hline 3 & 1 & 2 & 50.00 \\
\hline 4 & 7 & 5 & -36.36 \\
\hline 5 & 1 & 0 & -66.67 \\
\hline 6 & 10 & 33 & 241.38 \\
\hline 7 & 4 & 6 & 72.73 \\
\hline 8 & 3 & 2 & -44.44 \\
\hline 9 & 382 & 457 & 19.44 \\
\hline 10 & 414 & 24 & -94.13 \\
\hline 11 & 41 & 443 & 970.97 \\
\hline 12 & 433 & 446 & 3.00 \\
\hline 13 & 1 & 0 & -100.00 \\
\hline 14 & 360 & 282 & -21.69 \\
\hline 15 & 442 & 454 & 2.87 \\
\hline
\end{tabular}




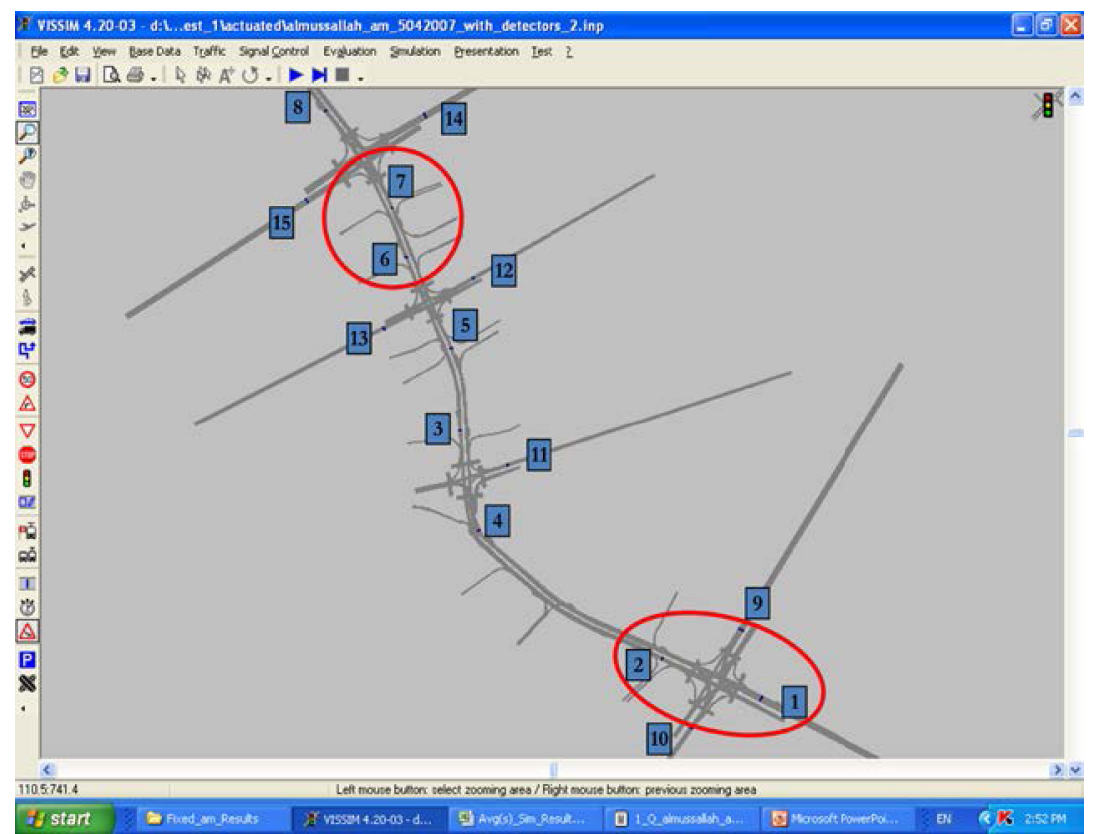

Fig. 9. Locations of the highest queue length (fixed v/s actuated).

In summary, this study investigated the impact of the TSP on Al Mussallah/ Al Maktoum corridor. Hence, Buses received reduction in travel time by $7.04 \%$ when actuating traffic signals. On the other hand, cars receive about $38.68 \%$ as an increase on its travel time. Also, buses receive delay reduction of about $14.11 \%$, whereas cars delay is increased by $57.00 \%$. Which is quite good compared to the international practices. For instant, active TSP improved bus operation through reduced average bus delay from 35.9 to 28.0 seconds (by $22.1 \%$ ) against no scenario as well as improved bus speed by $4.1 \%$ which improved bus performance as proved by (Lee \& Shalabi, 2011) [17] when they studied the strategy implementation at one of City of Brampton main streets which is crossing the city south-north with 2-lane per each direction at queensland- Australia.

In general; the Green extension and the Early green strategy (TSP actuated strategy), provide slight improvement in the bus route in term of travel time, delay, and stop points. However, there is still an increase in the queue length. (Table 5) shows that the higher the green extension, the better the results that are generated according to the common practices. It is most likely to introduce the TSP strategy at first in the off peak in order to evaluate the impact of it in the bus service since it normally receives more delays in the peak hours. Improving the headways also would generate better results as to improve the bus services. In addition, to dedicate a special lane for buses would also impact its travel time and headway since these lanes will not be competing with other traffic flows running through these intersections. 


\section{Conclusion}

\subsection{TSP impact summary}

TSP strategy also will essentially influence the public transport service in Dubai by the following:

- Extending the green time to accommodate bus movement though the signalized intersection. Since the study corridor contains 4 signalized intersections, then the bus route will be impacted positively in the whole study corridor because the green extension will be coordinated in the 4 intersections.

- Improving Buses reliability/ efficiency though adherence to transit schedules, leads private vehicle drivers to shift their mode of transport (model split) into PT.

- When private vehicle driver becomes a bus passenger, then he will gain peaceful time to relax, while the signal movements frustrate other private vehicle drivers.

- Because of the yield and drive status caused by the red truncation in the nontransit vehicle priority approaches, vehicles will reach the wear condition very fast. Thus, vehicle owners will suffer from regular maintenance money lose.

- In term of the environmental aspect, reducing number of private vehicles in a corridor will reduce vehicle fuel consumption and emissions.

\subsection{Dubai case study results}

Impacts of actuating traffic signals in Al Mussallah/ Al Maktoum corridor after extending green time for bus approach $(15 \mathrm{sec})$ :

- Minimum reduction occurs on buses average travel time along the study corridor by $7.04 \%$ when applying TSP.

- Total travel time of cars in the network will be tremendously increased by $38.68 \%$ when applying TSP.

- Reduction in cars average speed is received when applying TSP by $28.04 \%$. Whereas, buses average speed is increased by $35.52 \%$

- Reduction in buses delay is received when applying TSP by around $14.11 \%$. In addition, there is decreased average stopped delay per bus of about $18.56 \%$. On the other hand, cars received an increase in a delay about $57.00 \%$, with increased average stopped delay per car of about $55.13 \%$.

- Overall network performance for all vehicles is similar to the passenger car performance.

- TSP sometimes increases the queue length in links including bus routes, or it shows a reduction. On other links, the TSP in general increases queue lengths.

- As a result, public transport buses reliability and efficiency will be increased. This will lead to encourage people to shift their mode of transport.

- Overall conclusion is to achieve better rider-ship in Dubai.

\subsection{Recommendations}

It is worthwhile to consider that the transit vehicle (Buses) carries significantly higher occupancy than Private Vehicles (cars). A reasonable balance must be maintained between transit vehicles and other vehicles running in the corridor in terms of its travel time and delays in order to deliver a successful TSP strategy for all stakeholders involved in the implementation of this strategy. In other words, the private vehicle travelers might have 
other alternatives when they receive delays in travel time, so their decision might be challenging. As an example, they could choose a dynamical route as an alternative. Accordingly, this leads RTA to think more comprehensively about the TSP associated services. Then RTA is responsible to integrate the existing and the future public buses services with other transit services proposed by their studies such as the metro and ferry system. In addition, to improve the quality of the public bus service RTA is recommended to adopt the most recent intelligent transportation system such as TSP. However, to achieve better results when implementing TSP strategy, other aspects should be considered and integrated with the proposed strategy such as:

- Improving public bus system according to its frequencies and stop locations and integrate the full network in traffic signals integration system.

- Improving other intelligent transportation system-ITS incorporated with the Transit vehicles (i.e. GPS, Smart Phone Bluetooth, real-time systems .. etc).

- Considering the waiting time for transit vehicle passengers to ride in the bus stops by preparing adherence time tables for buses as well as shortening bus headways and frequencies running in the corridor (i.e. from every 20 minutes to 15 or less).

Signals data and intersection approaches are also recommended to be reviewed and evaluated under different traffic conditions (i.e. on/ off peak periods). Reviewing road networks and bus routes based on the data collection methodologies in different horizons such as surveys, interviews, technical standards and specifications is also recommended. This is needed in order to establish future guidance in UAE adaptation for some implemented successful established case studies for Transit and Transportation/ Traffic agencies which worked together to support local agencies when exploring the implementation of TSP. Overall Guideline for a successful implementation is when its considered in the Planning phase which will continuously strengthen and develop it during each project phase. Very advanced system and design of traffic signal cabinets allow for transit agencies to access the equipment. To encourage the involvement of all stakeholders in the TSP strategy, more reliable data collected with real-time platforms, and to carry on maximize financial benefit reports for all is recommended. Florida had the best practice on successful guidelines for the same (Diane Quigley, 2014) [18].

Although the capital costs to implement TSP per intersection will approximately cost a total of $(\$ 5,000)$, the cost of software installation is $(\$ 20,000)$ and controller equipment is $(\$ 30,000)$. It has a high impact and is recorded when it is implemented on projects planning stage as per Planning and Implementation Handbook, FTA [2].

Overall conclusion is if Dubai RTA considers all the above-mentioned recommendations then the rider-ship in Dubai will be improved and roads network congestion will be reduced.

\section{References}

1. S. R. Sunkari, Beasley, "Model to Evaluate the Impacts of Bus Priority on Signalized Intersections", P.S. Urbanik II, Transportation Research, (1995).

2. Garrow, M. and Machemehl, "Development and Evaluation of Transit Signal Priority Strategies", Research Report, SWUTC/97/472840-00068-1, Southwest Region University Transportation Center, Center for Transportation Research, University of Texas at Austin, (1998).

3. Peter Koonce, W. C. \& K. Turner "Implementing Traffic Signal Priority for A. Skabardonis A. Skabardonis A. Skabardonis A. Skabardonis A. Skabardonis Buses", Compendium of Technical Papers. Institute of Transportation Engineers, (1999). 
4. A. Skabardonis, "Control strategies for transit priority". Paper presented at the 79th Annual Meeting of the Transportation Research Board, (2000).

5. Manual of Uniform Traffic Control Devices, Millennium Edition, Federal Highway Administration, US, Dept. of Transportation, Washington DC, US, (2000).

6. Vahidi, H. "Transit Signal Priority: A Comparison of Recent and Future Implementations" Presented at 70th Annual ITE Meeting in Nashville, TN, (2000).

7. Westwood Professional Services, Inc. "St. Cloud Metropolitan Transit Commission Transit Priority Evaluation Project”, Final Report, prepared to St. Cloud Metropolitan Transit Commission, Minnesota, November (2000)

8. Kiel Ova, Ayman Smadi, "Evaluation of Transit Signal Priority Strategies for Small- Medium Cities", December (2001).

9. Washington State Transportation Centre (TRAC), "Transit Signal Priority System Performance Monitoring and Optimization", January (2001).

10. Ghada, S. and Newland, "Effectiveness of Bus Signal Priority", National Center for Transit Research, Center for Urban Transportation Research, University of South Florida, Tampa, FL, January (2002).

11. Synchro software program, User Guide 6, David Husch, John Albeck, Traffic-ware Publisher, (1993-2003)

12. VISUM, User Manual version 9.4, PTV Version, 2006

13. VISSIM, User Manual version 4.2, PTV Version, 2006

14. VisVAP, Appendix A: Add-On VAP, VISSIM Manual, PTV, VAP version 2.15, (2006)

15. VisVAP, Appendix B: Add-On VisVAP, VISSIM Manual, PTV, VisVAP version $2.15,(2006)$

16. TCRP Synthesis 83-bus and Rail Transit Preferential Treatments in Mixed use Traffic, Asynthesis of Transit Practice, Consultant Alan R.Danher, PB Americas, Inc. , Orlando - Florida, (2010)

17. Rule based Transit Signal Priority Control Method using a Real-Time Transit Travel Time Prediction Model Queensland university of Technology, Australia Lee, Jinwoo \& Shalaby, Amer , -, (2011)

18. Diane Quigley, Florida Dept. of Transportation, Transit Signal Priority Implementation Guidance, Final Report, July 7, (2014) 